\title{
The Effect of Bulgecin A on Peptidoglycan Metabolism and Physiology of Helicobacter pylori
}

\author{
Mathilde Bonis, ${ }^{1,2}$ Allison Williams, ${ }^{1,2}$ Stephanie Guadagnini, ${ }^{3}$ Catherine Werts, ${ }^{1,2}$ and Ivo G. Boneca ${ }^{1,2}$
}

Helicobacter pylori, a human-specific bacterial pathogen responsible for severe gastric diseases, constitutes a major public health issue. In the last decade, rates of $H$. pylori resistance to antibiotics were increasing drastically, requiring alternative therapeutic strategies to deal with eradication failures. Therefore, we evaluated the potential of bulgecin A, a glycosidic inhibitor of the lytic transglycosylase (LTG) Slt70 of Escherichia coli, as a new therapeutic approach against the H. pylori infection. In this study, we show that bulgecin A is able to specifically inactivate the H. pylori LTG Slt, but not its ortholog MltD. Moreover, bulgecin A synergized with amoxicillin, an inhibitor of penicillin binding proteins, inducing strong morphological alterations, cellular damages, and cell death. Similarly, the simultaneous inactivation of the peptidoglycan (PG) peptidase HdpA and Slt led to inhibition of $H$. pylori growth, highlighting the strong potential of targeting the PG biosynthetic pathway at different biochemical steps to enhance our therapeutic approaches against bacteria. Hence, we propose that bulgecin A constitutes an attractive compound for the development of new therapeutic strategies against $H$. pylori combined with other inhibitors of PG biosynthetic enzymes.

\section{Introduction}

H ELICOBACTER PYLORI (H. PYLORI) is a major human pathogen that infects nearly half the world's population, and is linked to serious gastric diseases. Indeed, it is now established that the $H$. pylori infection is responsible for most of the cases of ulcerative diseases, gastric MALT lymphoma, and gastric adenocarcinoma. ${ }^{17}$ This latter constitutes the second cause of cancer-related mortality, leading to 700,000 deaths per year throughout the world. ${ }^{30}$ Nowadays, the treatment of $H$. pylori infection mostly relies on the association of several molecules, such as proton pump inhibitors, amoxicillin, and/or clarithromycin. However, these antibiotics have to cope with the increasing rates of bacterial resistances, responsible for therapeutic failure, arising in approximately a third of patients. Additionally, no commercialized vaccine against this bacterium is currently available. Therefore, $H$. pylori infection still constitutes an important public health issue, requiring the identification of new therapeutic strategies.

In that context, the study of the peptidoglycan (PG) metabolism of $H$. pylori might provide some precious clues for the discovery of new therapeutic targets. Indeed, by forming a mesh network around the bacteria, PG plays a crucial role to counteract the osmotic pressure and for the bacterial morphogenesis. It is composed of glycan chains of repeating disaccharide units of $\mathrm{N}$-acetylglucosamine (GlcNAc) and $\mathrm{N}$ acetylmuramic acid (MurNAc) crosslinked via peptide bonds. Throughout the cell cycle, this molecule requires the intervention of different proteins, including enzymes able to digest old PG and others able to assemble the PG precursors, allowing for the bacterial elongation and division. Hence, the inactivation of such proteins can seriously impact on cell viability, especially in $H$. pylori that has a relative reduced genome with poor redundancy of protein functions. Several studies have recently highlighted the function of some of these enzymes. First, Chaput and colleagues characterized three hydrolases: the putative amidase AmiA, and the lytic transglycosylases (LTGs) MltD and Slt. It has been shown that cell separation and morphological transformation of H. pylori into coccoid bacteria required the activity of AmiA. ${ }^{4}$ Otherwise, Slt and MltD are both nonredundant LTGs, capable of cleaving the PG glycan chains in an exo and an endo manner, respectively, generating anhydromuropeptides. ${ }^{5}$ Moreover, Slt participates in the inflammatory response during $H$. pylori infection by releasing a Nod1 ligand (the GMtripeptide, GM3), leading to the activation of NF- $\kappa \mathrm{B}$, and, consequently, to the production of the proinflammatory

\footnotetext{
${ }^{1}$ Institut Pasteur, Group Biology and Genetics of the Bacterial Cell Wall, Paris, France.

${ }^{2}$ INSERM, Groupe Avenir, Paris, France.

${ }^{3}$ Institut Pasteur, Plate-Forme de Microscopie Ultrastructurale, Paris, France.
} 
cytokine IL8. ${ }^{28}$ Second, genome analysis revealed the presence of three high-molecular-weight penicillin-binding proteins (PBPs), whereas $H$. pylori genome was intriguingly lacking low-molecular-weight PBPs. ${ }^{2,7}$ Nevertheless, recent studies described the activity of a new enzyme (HdpA/ Csd3) that might compensate, at least partially, this absence $^{3,23}$ Indeed, contrary to the other members of the M23 family, HdpA carries both D,D-carboxy and -endopeptidases activities. Additionally, these activities are involved in the $H$. pylori ability to colonize the mouse gastric mucosa, presumably due to their role in the regulation of the bacterial $\mathrm{rod} /$ spiral shape.

Hence by seeking a way to inactivate PG-related enzymes in H. pylori, we decided to focus our work on bulgecin A, previously described in the literature as an inhibitor of Slt70 from Escherichia coli. ${ }^{24}$ Originally, this molecule was purified in the 1980s from the species Pseudomonas acidophila and characterized by the generation of bulges in E. coli grown in presence of $\beta$-lactams. ${ }^{13}$ Since, it has been shown, by both enzymatic assays and X-ray crystallographic analysis, that bulgecin A could bind and inactivate the catalytic domain of Slt70..$^{21,25}$ In fact, bulgecin $\mathrm{A}$ is a derivative of the GlcNAc, mimicking the PG natural substrate of Slt70. As Slt from H. pylori and Slt70 share a relative important degree of homology on their catalytic domains (around 30\% of amino acids identity), we asked whether bulgecin A could be active on the PG metabolism of $H$. pylori. In this work, we show that bulgecin A induced the same PG modifications as those exhibited by the mutant slt. In addition, by inhibiting the release by Slt of the proinflammatory GanhMtripeptide, bulgecin A is able to reduce the inflammatory response to $H$. pylori in an in vitro model of HEK293T cells. Moreover, 3D structural prediction of Slt and MltD in the presence of bulgecin A provided a model for the specificity of bulgecin A activity toward Slt. We also showed that bulgecin A was able to act synergistically with amoxicillin (an inhibitor of PBP), leading to morphological abnormalities and a loss of viability. Despite a milder effect, this synergy seemed also active on the H. pylori colonization of the mice gastric mucosa. Finally, we noticed that the impact of bulgecin A on the growth of $H$. pylori was strongly increased when $h d p A$ was simultaneously deleted. Thus, we show in this work that bulgecin A may constitute an attractive therapeutic agent, since it is able to target the LTG Slt in $H$. pylori, leading to an increased sensitivity to the inactivation of several PG-related enzymes.

\section{Materials and Methods}

\section{Bacterial strains and growth conditions}

H. pylori strains and mutants (listed in Table 1) were grown on blood agar plates, or in liquid culture in BHI supplemented with $10 \%$ of decomplemented fetal calf serum (FCS) and an antibiotic-antifungal $\mathrm{mix}\left(0.31 \mu \mathrm{g} \cdot \mathrm{ml}^{-1}\right.$ of polymyxin B, $2.5 \mu \mathrm{g} \cdot \mathrm{ml}^{-1}$ of amphotericine $\mathrm{B}, 12.5 \mu \mathrm{g} \cdot \mathrm{ml}^{-1}$ of vancomycin, and $6.25 \mu \mathrm{g} \cdot \mathrm{ml}^{-1}$ of trimethoprim) and appropriated antibiotic $\left(20 \mu \mathrm{g} \cdot \mathrm{ml}^{-1}\right.$ of kanamycin), at $37^{\circ} \mathrm{C}$ in a microaerophilic atmosphere. For some experiments, bacteria were grown in the presence of $100 \mu \mathrm{g} \cdot \mathrm{ml}^{-1}$ of bulgecin A (provided by Takeda Pharmaceutical Company Ltd.) ${ }^{13}$ or $0.02 \mu \mathrm{g} \cdot \mathrm{ml}^{-1}$ of amoxicillin.
Table 1. Helicobacter pylori Strains and Mutants with Respective References, Used for the Present Study

\begin{tabular}{|c|c|}
\hline Strain & Reference \\
\hline 26695 & Tomb et al. ${ }^{26}$ \\
\hline 26695 slt $^{-}$ & Chaput et al. ${ }^{5}$ \\
\hline $\mathrm{X} 47$ & Ermak et al. $(1998)^{8}$ \\
\hline $\mathrm{X} 47 \mathrm{mlt} D \Omega \mathrm{Km}$ & Roure $^{20}$ \\
\hline $\mathrm{X} 47$ slt $\Omega \mathrm{Km}$ & Roure $^{20}$ \\
\hline $\mathrm{X} 47 \mathrm{hdp} A \Omega \mathrm{Km}$ & Bonis et al. ${ }^{3}$ \\
\hline N6 & Ferrero et al. (1992) \\
\hline
\end{tabular}

\section{Muropeptides composition analysis}

The PG from strain 26695 grown in the presence or not of $100 \mu \mathrm{g} \cdot \mathrm{ml}^{-1}$ of bulgecin A was extracted from 3 distinct cultures of $100 \mathrm{ml}$ stopped in exponential growth $\left(\mathrm{OD}^{600 \mathrm{~nm}}\right.$ around 0.8 ) in an ice-ethanol bath. The crude sacculi were immediately extracted by boiling for $20 \mathrm{~min}$ in SDS ( $4 \%$ final concentration). After cooling, the SDS was removed by several ultracentrifugation $\left(39,000 \mathrm{rpm}, 30 \mathrm{~min}, 18^{\circ} \mathrm{C}\right)$ by washing with distilled water. The total elimination of the SDS was checked as described. ${ }^{11}$ Fifty microliters of each PG suspension $\left(1 \mathrm{mg} \cdot \mathrm{ml}^{-1}\right)$ was digested by 80 units of mutanolysin (Sigma-Aldrich) in $12.5 \mathrm{mM}$ of sodium phosphate buffer $\mathrm{pH} 5.8$ (in a final volume of $200 \mu \mathrm{l}$ ), overnight at $37^{\circ} \mathrm{C}$. Samples were then reduced with $0.5 \mathrm{M}$ of borate buffer $\mathrm{pH} 9$ (volume to volume) to which $\sim 5 \mathrm{mg}$ of sodium borohydride was added as a catalyzer for $30 \mathrm{~min}$. The reaction was stopped by adjusting the final $\mathrm{pH}$ to 2 with 10 to $20 \mu \mathrm{l}$ of a $20 \%$ orthophosphoric acid solution. Muropeptides were analyzed by HPLC using a Hypersil ODS18 reverse-phase column with a $0 \%$ to $15 \%$ methanol gradient in sodium phosphate buffer ( $\mathrm{pH} 4.3$ to 4.9 ). ${ }^{10}$ Chromatograms were obtained by monitoring of muropeptides elution at $206 \mathrm{~nm}$, and relative area of each peak was estimated using LabSolution software (Shimadzu). The respective glycan chain length averages were calculated as previously described. ${ }^{10}$ Data are presented in means \pm SEM, obtained from three distinct cultures.

\section{HEK293T cells cotransfection}

H. pylori strain 26695 was grown in liquid BHI supplemented with $10 \%$ of FCS in the presence or absence of bulgecin A $\left(100 \mu \mathrm{g} \cdot \mathrm{ml}^{-1}\right)$. As previously described ${ }^{29}$ human HEK293T cells, grown in 24-well plates in $500 \mu$ l of DMEM, were transiently cotransfected with a human Nod1 expression vector $(1 \mathrm{ng})$ together with an NF-kB luciferase-reporter construct (75ng), and a $\beta$ galactosidase-expressing vector $(25 \mathrm{ng})$, using Fugene reagent. Thirty minutes before adding the DNA complexes, $20 \mu \mathrm{l}$ of culture supernatants, from bacteria grown for $12 \mathrm{hr}$ and stopped by centrifugation when $\mathrm{OD}^{600 \mathrm{~nm}}$ reached 1, was added to the cells. As controls, MurTriDap (MTP; $50 \mathrm{nM})$, MDP $(50 \mathrm{nM})$, or $20 \mu \mathrm{l}$ of sterile bacterial growth medium was used. After $24 \mathrm{hr}$, cells were lysed, and both luciferase and $\beta$ galactosidase activities were measured. Results are presented as relative luciferase units (RLU) normalized to $\beta$ galactosidase activity. Data show the mean \pm SEM of triplicates and are representative of three independent experiments. 


\section{Homology modeling of Slt and MItD}

Phyre (www.sbg.bio.ic.ac.uk/ phyre/secretindex.cgi), the protein homology recognition server, was used to select the best three-dimensional structure for modeling Slt and MltD. Slt70 (PDB:ID 1SLTY) was selected as the best fit model. Using the Xray structure of Slt70 as a template, models for Slt and MltD were generated by the program MOLREP. The models were visualized and evaluated using PYMOL. Structural figures were generated using PYMOL.

\section{Kinetic growth}

Kinetics of growth were performed by following the viability of $H$. pylori strains X47, X47slt $2 \mathrm{Km}$ (18), and N6. Bacteria were grown in liquid culture in the presence of $100 \mu \mathrm{g} \cdot \mathrm{ml}^{-1}$ of bulgecin A, $0.02 \mu \mathrm{g} \cdot \mathrm{ml}^{-1}$ of amoxicillin, or the association of both molecules, during $47 \mathrm{hr}$. For each time point, suspensions were diluted and then spread on blood agar plates (see Bacterial strains and growth conditions section). After 3 days of incubation at $37^{\circ} \mathrm{C}$, colony forming

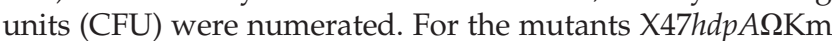
and $\mathrm{X} 47 \mathrm{mlt} \mathrm{D} \Omega \mathrm{Km}$, kinetic curves were achieved by measuring the absorbance at $600 \mathrm{~nm}$ of the bacterial cultures during $47 \mathrm{hr}$.

\section{Scanning electron microscopy}

The morphology of the strain X47 grown with $100 \mu \mathrm{g} \cdot \mathrm{ml}^{-1}$ of bulgecin A, $0.02 \mu \mathrm{g} \cdot \mathrm{ml}^{-1}$ of amoxicillin, or the association of both molecules was analyzed by scanning electron microscopy. Bacteria from overnight cultures were fixed in $2.5 \%$ glutaraldehyde in $0.1 \mathrm{M}$ cacodylate buffer ( $\mathrm{pH}$ 7.2). They were then washed three times for $5 \mathrm{~min}$ in $0.2 \mathrm{M}$ cacodylate buffer ( $\mathrm{pH} 7.2$ ), postfixed for $1 \mathrm{hr}$ in $1 \%$ (wt/vol) osmium tetroxide in $0.2 \mathrm{M}$ cacodylate buffer $(\mathrm{pH} 7.2)$, and then rinsed with distilled water. Samples were dehydrated through a graded series of $25 \%, 50 \%, 75 \%$, and $95 \%$ ethanol solution for $5 \mathrm{~min}$ (each time). Samples were then dehydrated for $10 \mathrm{~min}$ (each time) in 100\% ethanol followed by critical-point drying with $\mathrm{CO}_{2}$. Dried specimens were sputtered with 10-nm goldpalladium with a GATAN Ion Beam Coater and were examined and photographed with a JEOL JSM 6700F field emission scanning electron microscope operating at $5 \mathrm{kv}$. Images were acquired with the upper SE detector (SEI).

\section{Mice experiments}

Six-week-old female mice (C57BL/6J; Charles River) were infected with $\mathrm{X} 47$ by oral route with feeding needles $\left(2 \times 10^{8}\right.$ bacteria per mouse in $200 \mu \mathrm{l}$ of peptone broth). After 7 days of infection, mice were treated by oral route according to two protocols: for conditions 1 to 4 , mice received, once a day, distilled water as a negative control, $20 \mu \mathrm{g}$ of bulgecin A, $2 \mathrm{mg}$ of amoxicillin, or the association of both molecules in a final volume of $200 \mu \mathrm{l}$. For the last two conditions, 5 and 6, mice received, twice a day, $0.1 \mathrm{mg}$ of amoxicillin alone or associated with $20 \mu \mathrm{g}$ of bulgecin A, respectively. Eight mice were treated for each condition. After 7 days of treatment, mice were euthanized with $\mathrm{CO}_{2}$ and the stomachs were ground and homogenized in peptone broth. The samples were then diluted and spread on blood agar plates supplemented with $200 \mu \mathrm{g} \cdot \mathrm{ml}^{-1}$ of bacitracin and $10 \mu \mathrm{g} \cdot \mathrm{ml}^{-1}$ of nalidixic acid to inhibit the growth of resident bacteria from the mouse forestomach. The CFUs were enumerated after 8 days of incubation.

\section{Statistics}

The statistical analysis of the mouse experiments were performed using Graph Pad Prism software, with a twotailed nonparametric Mann-Whitney test. One asterisk corresponds to a $p$-value falling between 0.05 and 0.01 , and three asterisks correspond to a $p$-value less than 0.001 .

\section{Results}

\section{Effect of bulgecin A on the muropeptide composition of $\mathrm{H}$. pylori}

Since bulgecin A has been described in the literature as a specific inhibitor of the LTG Slt70 of E. coli, ${ }^{24}$ we first decided to evaluate the impact of bulgecin A on the H. pylori PG composition. We therefore purified the sacculi from cultures of strain 26695 grown in the presence or absence of bulgecin A. PG was then digested with mutanolysin, a muralytic enzyme that cleaves the MurNAc-( $\beta 1-4)-G l c N A c$ linkage, to estimate by HPLC the relative proportion of each muropeptide (Fig. 1). First, our data showed that the presence of bulgecin $\mathrm{A}$ in the growth medium was correlated to a significant decrease of the anhydromuropeptides rate, these moieties being the result of LTG activity. In addition, bulgecin A led to the accumulation of the muropeptide GM3, associated with the enhancement of the glycan chain length average. Interestingly, these two PG modifications have been previously described for the mutant lacking slt. ${ }^{5}$ Contrary to this latter, the mutant 26695 mltD does not present any significant muropeptide composition modification, while the double-mutant 26695 slt/mltD exhibits a proportion of anhydromuropeptides strongly reduced compared to 26695 slt. ${ }^{5}$ Altogether, these results indicated that bulgecin A could target and specifically inactivate Slt.

\section{Bulgecin A modifies the inflammatory response to $\mathrm{H}$. pylori in vitro}

To confirm the inhibitory activity of bulgecin A on Slt, we focused on another aspect of the activity of this hydrolase. Indeed, Slt participates in the inflammatory response due to H. pylori by releasing a Nod1 ligand (the GanhM3), responsible for stimulating NF- $\mathrm{kB}$, and consequently for the synthesis of proinflammatory cytokines such as IL8. ${ }^{28}$ Hence, we addressed the question whether bulgecin A could interfere with this inflammatory response. We stimulated HEK293 cells with supernatants of cultures of $H$. pylori strain 26695, grown in presence or absence of bulgecin A, just before the cotransfection of cells with a Nod1 and an NF-кB luciferasereporter expression plasmids (Fig. 2). By this way, muropeptides released in the culture supernatants entered the cells with the transfection reagent. The light signal obtained is proportional to the stimulation of Nod1 by the GanhM3. As negative controls, we used the Nod2 ligand MDP, the sterile growth medium, and supernatants of cultures of the mutant 26695 slt. The MTP was used as a positive control. The data obtained revealed that the presence of bulgecin $\mathrm{A}$ in the growth medium of the 26695 strain led to a reduced number of relative light units (RLU) compared to the supernatants of the wild type, to the same extent than the 


\begin{tabular}{|c|c|c|}
\hline Muropeptides & 26695 & $26695+$ bulgecin $A$ \\
\hline GM3 (1) & $10,5^{+/-0.6}$ & $16,0^{+1-0.4}$ \\
\hline GM4 (2) & $5,9^{+/-0.6}$ & $4,1^{+1-0.5}$ \\
\hline GM4gly (3) & $3,1^{+1-0.2}$ & $2,8^{+/-0.2}$ \\
\hline GM2 (4) & $1,8^{+/-0.1}$ & $2,1^{+/-0.1}$ \\
\hline GM5 (5) & $33,5^{+/-0.4}$ & $34,4^{+1-1.7}$ \\
\hline GM4-3 (6) & $5,9^{+1-0.2}$ & $7,4^{+1-0.8}$ \\
\hline GM4gly-4 (7) & $3,6^{+1-0.1}$ & $3,1^{+/-0.1}$ \\
\hline GM4-4 (8) & $5,3^{+/-0.3}$ & $4,4^{+/-0.2}$ \\
\hline GMS-4 (9) & $17,3^{+/-0.8}$ & $16,0^{+/-0.9}$ \\
\hline GanhM5 (10) & $1,6^{+/-0.4}$ & $1,6^{+/-0.2}$ \\
\hline GanhM4-3GM (11) & $3,1^{+1-0.5}$ & $3,4^{+1-0.4}$ \\
\hline GanhM4-4GM (12) & $2,8^{+/-0.1}$ & $1,4^{+/-0.4}$ \\
\hline GanhM5.4GM (13) & $5,6^{+1-0.7}$ & $3,3^{+/-0.8}$ \\
\hline Monomeres (14) & $56,2^{+/-0.6}$ & $61,0^{+/-1.3}$ \\
\hline Dimeres (15) & $43,8^{+/-0.6}$ & $39,0^{+/-1.3}$ \\
\hline Anhydromuropeptides (16) & $13,2^{+/-1.0}$ & $9,7^{+/-1.3}$ \\
\hline Glycan chain length average & $10,9^{+/-0.8}$ & $14,5^{+/-1.9}$ \\
\hline
\end{tabular}

B
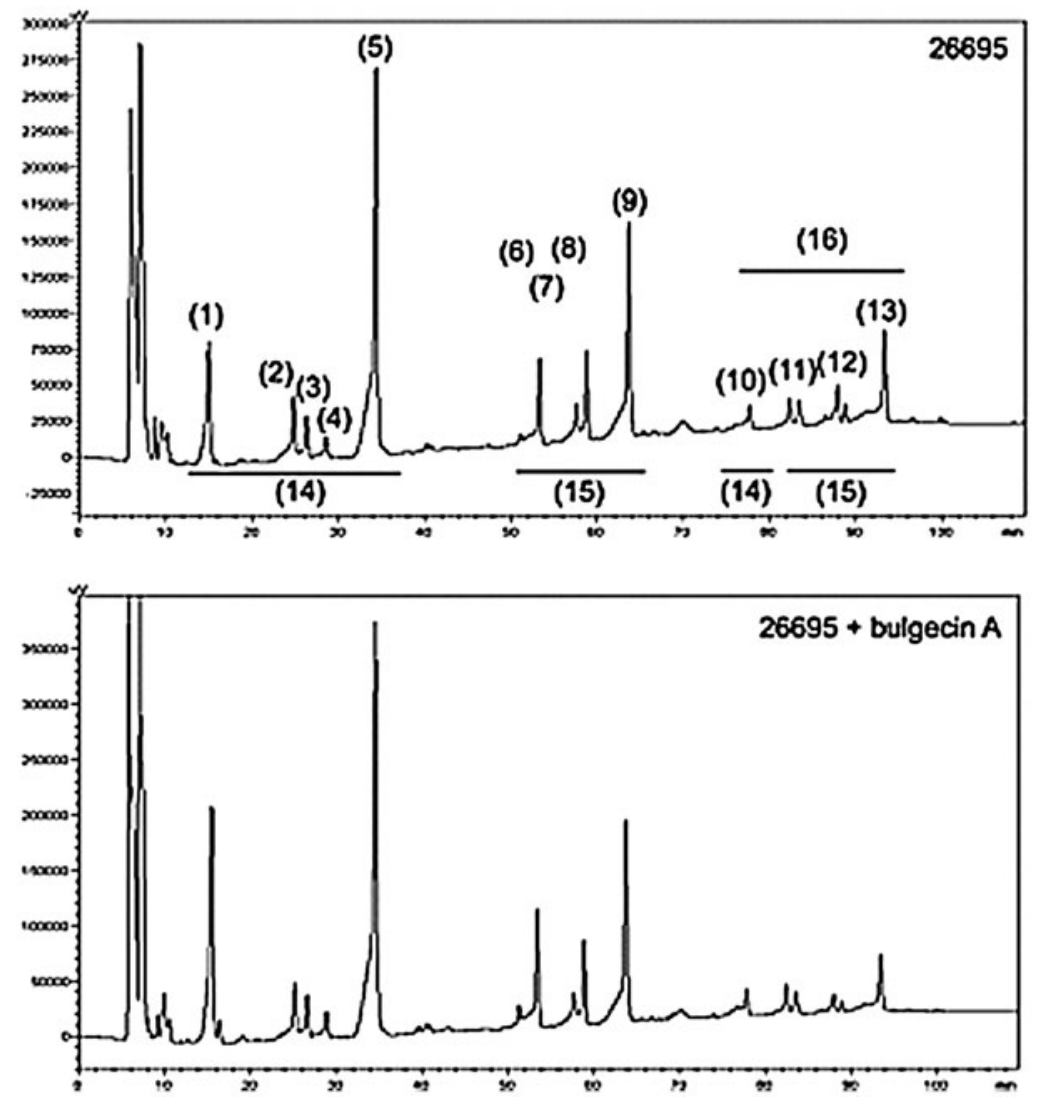

FIG. 1. Muropeptides composition analysis of Helicobacter pylori incubated with bulgecin A. (A) Strain 26695 was grown in liquid medium supplemented or not with bulgecin A $\left(100 \mu \mathrm{g} \cdot \mathrm{ml}^{-1}\right)$. PG was extracted from bacteria in exponential growth phase and then digested by mutanolysin to estimate the relative proportion of each muropeptide. Data were analyzed from 3 distinct cultures, and values are expressed in means \pm SEM. The abbreviations are defined as followed: G, Nacetylglucosamine; M, N-acetylmuramic acid; anhM, N-acetyl-anhydromuramic acid. The numbers indicate the length of the stem peptide: 3 stands for the L-alanyl- $\gamma$-D-glutamyl-meso-diaminopimelic acid, 4 stands for the 3 peptide with an additional D-alanine, and 5 stands for the 3 peptide with an additional D-alanyl-D-alanine dipeptide. (B) Chromatographs of 26695 muropeptides in the absence or presence of bulgecin A. Each muropeptide has been numbered and referenced in A. PG, peptidoglycan. 


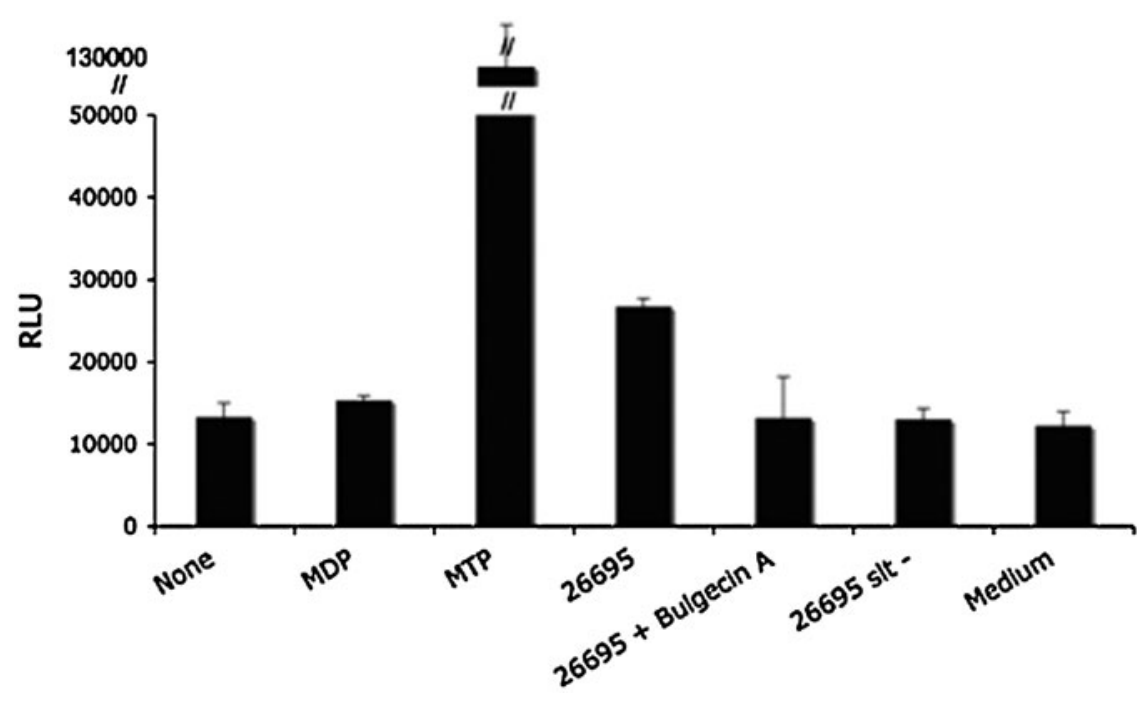

FIG. 2. In vitro effect of bulgecin A on the inflammatory response due to H. pylori. Human HEK293T cells were transiently cotransfected with a human Nod1 expression vector (1 ng), an NF- $\mathrm{B}$ luciferase-reporter construct (75 ng) and a $\beta$ galactosidase expressing vector $(25 \mathrm{ng})$. Thirty minutes before adding the DNA complexes, $20 \mu \mathrm{l}$ of culture supernatants, from 26695 slt $\Omega \mathrm{Km}$ or 26695 grown with or without bulgecin A, were added to the cells. MTP (MurTriDAP), MDP, and sterile bacterial growth media were used as controls. The graph represents the corresponding relative light units (RLU) normalized to the $\beta$ galactosidase activity. Data show the means \pm SEM of triplicates and are representative of three independent experiments.

deletion of slt (26695 slt). Thus, these results show that bulgecin A can affect the inflammation induced by 26695 in vitro, by inactivating the hydrolase Slt.

\section{Prediction of Slt and MItD binding to bulgecin A}

As our PG analysis predicted a specific activity of bulgecin A on the LTG Slt, we tried to understand why this molecule would not be able to target at the same time the other LTG from $H$. pylori, MltD. To answer that question, we established predictions of the 3D structures of Slt and MltD with bul- gecin A based on the available structures of Slt70 from E. coli crystallized with or without bulgecin A, and using the software MOLREP ${ }^{25,27}$ (Fig. 3). Our model showed that both LTGs would be formed by a large ring-shaped alphasuperhelix with on top a catalytic domain where bulgecin A is predicted to localize, in the same manner than for Slt70 (Fig. 3A). Interestingly, although the glutamic acid catalytic residue was strictly conserved, Slt exhibited an extended Cterminal domain with an additional helix corresponding to residues 556-566 in the catalytic domain, which was missing from MltD (Fig. 3B). Due to its location closed to the bulgecin
A

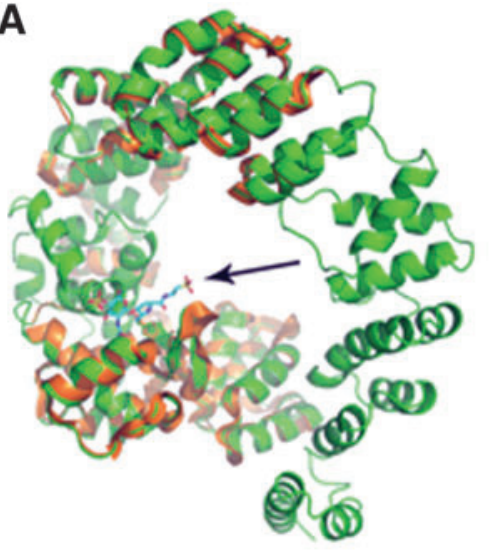

SIt MItD

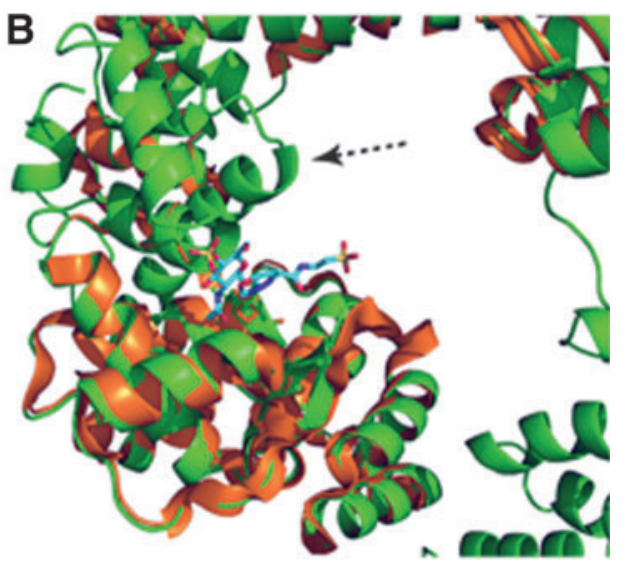

FIG. 3. Superimposition of Slt and MltD structural models with bulgecin A. Based on the available structures of Slt70 of E. coli, the 3D structures of Slt (green) and MltD (orange) were predicted and compared together. (A) depicts the whole structures and (B) is an enlargement of catalytic domains where bulgecin A is predicted to bind. Bulgecin A is indicated by the full arrow. The dotted arrow indicates the presence of an extra helix present in Slt, but absent in MltD. Figures have been drawn using the programs MELROP and PYMOL. 
A

X47

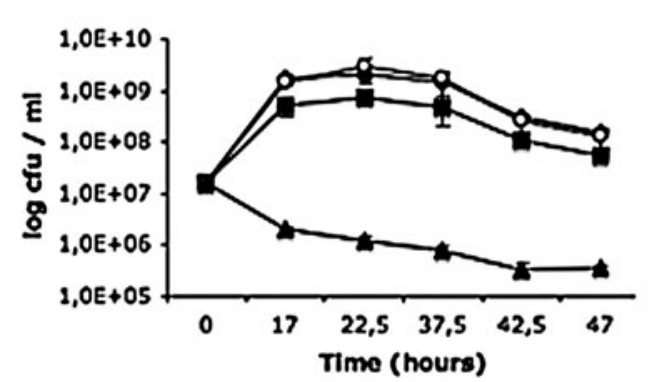

C

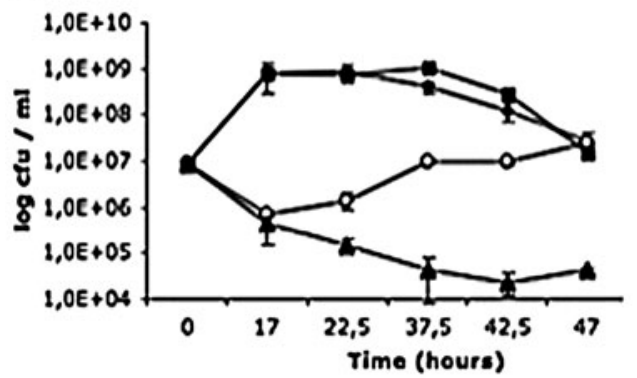

B

N6

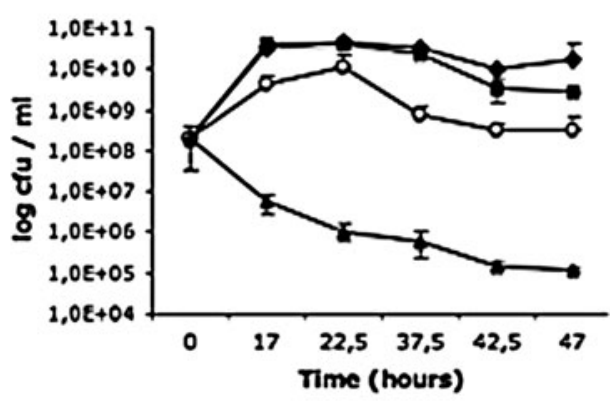

FIG. 4. Synergic activity of bulgecin A and amoxicillin on the H. pylori growth. The viability of strains X47 (A) and N6 (B), and the mutant $\mathrm{X} 47$ slt $\Omega \mathrm{Km}$ (C) were followed during $47 \mathrm{hr}$ in liquid medium (full diamond) supplemented with bulgecin A (full square), amoxicillin (sub-inhibitory concentrations (open circle)), or a mix of both molecules (full triangle). For each time point, triplicates of bacterial suspensions were diluted and spread on blood agar plates.
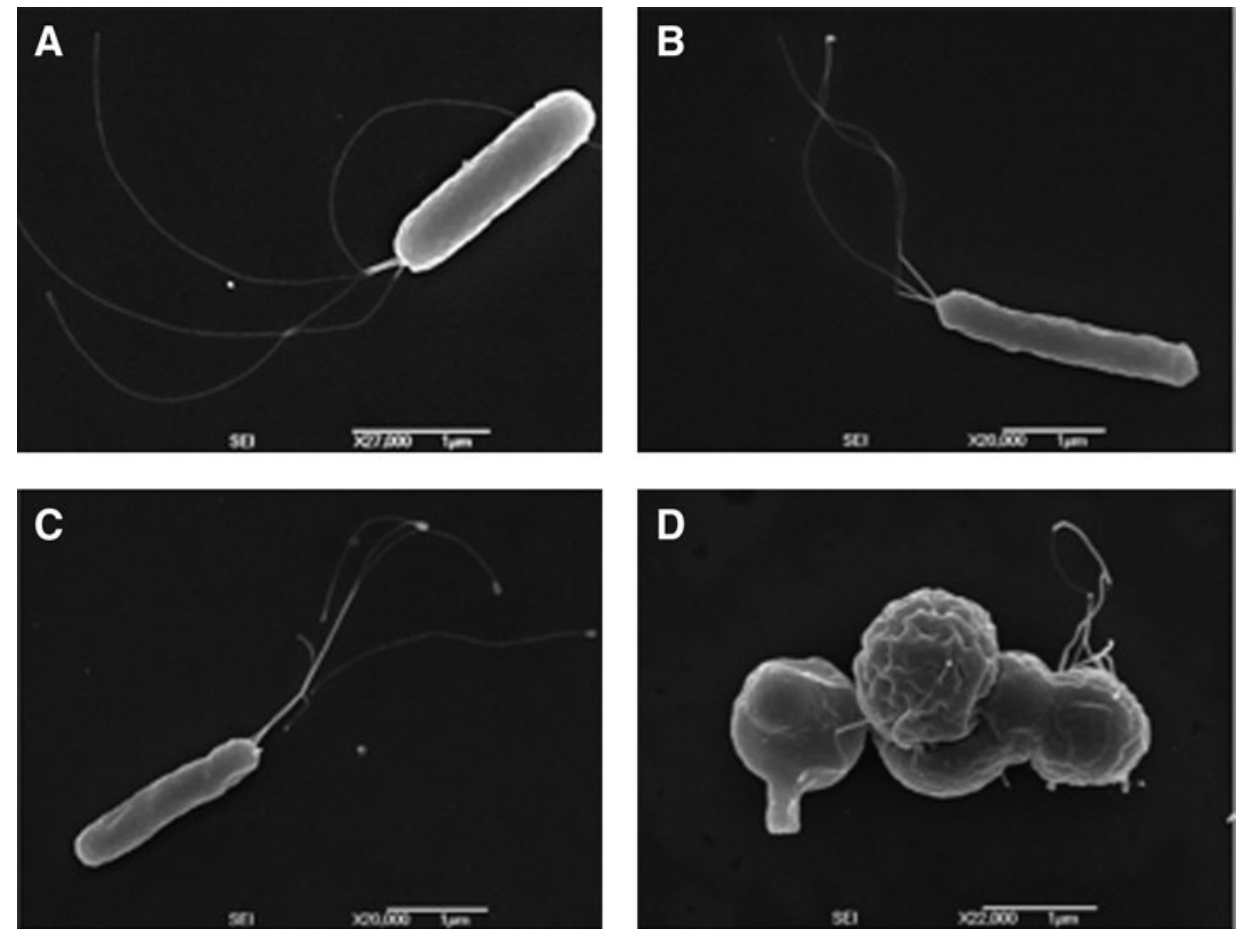

FIG. 5. Impact of bulgecin A and amoxicillin on bacterial morphology. The morphology of strain X47 was observed by scanning electron microscopy after $12 \mathrm{hr}$ of culture in liquid medium (A), supplemented with bulgecin A (B), amoxicillin (C), or a mix of both molecules (D). 
A binding site, this helix could play an important role for the proper positioning of bulgecin A and could be important for the Slt selectivity for bulgecin A.

\section{Synergistic activity of bulgecin A associated with amoxillin on the growth of $\mathrm{H}$. pylori}

Altogether, these previous data showed that bulgecin A was able to inhibit the activity of Slt in $H$. pylori. Since Slt plays a major role in the PG remodeling of $H$. pylori, we wondered if bulgecin A could be a new therapeutic candidate against this bacterial infection. However, from previous observations we noticed that bulgecin A used alone did not have any effect on the bacterial growth or morphology. Thus, we tried to optimize its potency by inactivating simultaneously another enzyme involved in PG metabolism. Here, we tested the effect of bulgecin A on the growth of several strains of $H$. pylori, in presence of a sub-inhibitory concentration of amoxicillin, this latter being able to inhibit the activity of PBPs (Fig. 4). By following the cell viability in liquid culture during $47 \mathrm{hr}$, we showed that bulgecin $\mathrm{A}$ and amoxicillin used, respectively, alone had a slight or no impact on the growth of X47 (Fig. 4A) and N6 (Fig. 4B). In contrast, the combination of both molecules induced cell death, associated with a strong growth decline. This suggests that bulgecin A and amoxicillin are able to act synergistically on the growth of $H$. pylori. Additionally, we reproduced this experiment with the mutant $X 47$ slt $\Omega \mathrm{Km}$ (Fig. 4C). Our data revealed that the presence of amoxicillin alone in the medium induced a strong decrease of cell viability, in particular for the early time points, while the growth of the parental strain was intact in these conditions. This suggests that the synergy highlighted above is indeed linked to the inactivation of Slt by bulgecin A. Moreover, this synergy seems efficient in different $H$. pylori strains, since we also observed this activity in strains B128, 26695, and S107 (data not shown). This latter has been isolated from a human gastric biopsy and displays a reduced susceptibility to amoxicillin.

\section{Synergistic activity of bulgecin A coupled with amoxillin induces strong morphologic abnormalities in $\mathrm{H}$. pylori}

To further characterize the synergic activity of bulgecin A and amoxicillin, we evaluated its impact on $H$. pylori morphology. Thus, we incubated the strain X47 with bulgecin A, sub-inhibitory amount of amoxicillin, or a mix of these substances. After $12 \mathrm{hr}$ of growth in liquid cultures, morphology of bacteria was examined by scanning electron microscopy (Fig. 5). Pictures revealed that bacteria grown under pressure of both molecules (Fig. 5D) displayed strong morphological defects characterized by the emergence of large ovoid and irregular shapes. Conversely, bulgecin A and amoxicillin used, respectively, alone did not alter the morphology of X47 (Fig. 5B and C, respectively), compared to the negative control (Fig. 5A). Same observations have been done with the strain S107 (data not shown).

\section{Effect of bulgecin A and amoxicillin on the $\mathrm{H}$. pylori gastric mice colonization}

Since the synergy between bulgecin A and amoxicillin induces strong damages in H. pylori, we wanted to know if this association could constitute an alternative therapeutic approach against this infection. To answer that question, we tested the impact of these molecules on the colonization of the gastric mucosa, in the mouse model. Mice were infected by strain $X 47$ and, 7 days postinfection, were treated by oral route, once a day, with amoxicillin, bulgecin $\mathrm{A}$, or a mix of both molecules (Fig. 6). After 7 days of treatment, colonization rates were measured by numerating the CFUs per gram of stomach. In these conditions, our data revealed that the treatment with bulgecin A did not interfere with the colonization ability of X47, while amoxicillin significantly reduced the colonization rate. Nevertheless, the experiment allowed to highlight a slight but significant decrease of the amount of bacteria in stomach of mice that received the mix of bulgecin A and amoxicillin compared to the mice that were treated only with amoxicillin. Trying to optimize this effect, we also administered to the mice a smaller amount of amoxicillin but twice a day, as required by a classical amoxicillin treatment. Compared to the previous experiment, results showed a higher rate of colonization for mice that received amoxicillin, and a same extent of decrease between these latter and the mice treated with the mix. Therefore, these data show that bulgecin A and amoxicillin act synergistically in vivo, allowing a slight but reproducible

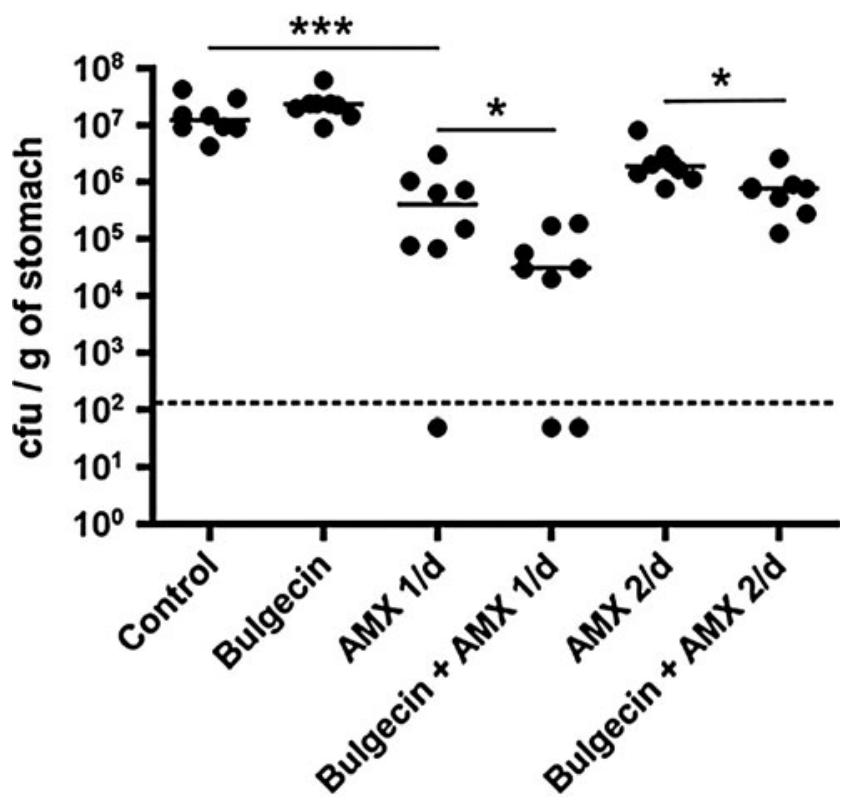

FIG. 6. Treatment of mice infected by H. pylori with bulgecin A and amoxicillin. C57BL/6J mice were infected orally by strain X47. Seven days postinfection, mice were treated by oral feeding according to two protocols. In conditions 2 to 4, mice received $20 \mu \mathrm{g}$ of bulgecin $\mathrm{A}, 2 \mathrm{mg}$ of amoxicillin, or a mix of equivalent amount of these molecules, for a final volume of $200 \mu \mathrm{l}$, once a day. The control (condition 1) corresponds to the administration of peptone broth, following the same schema. Mice of conditions 5 and 6 were treated twice a day with $0.1 \mathrm{mg}$ of amoxicillin associated or not with $20 \mu \mathrm{g}$ of bulgecin $\mathrm{A}$, in a same volume. After 7 days of treatment, mice were euthanized, and bacteria present in their stomach were numerated on blood agar plates. The full bars indicate the median of colony forming unit per gram of stomach for 8 mice $\left({ }^{* * *} p\right.$-value $<0.001 ;{ }^{*} 0.01<p$-value $<0.05$; Mann-Whitney test). The dotted bar represents the detection limit calculated for this experiment. 
decrease of $H$. pylori gastric colonization after 7 days of treatment. Nevertheless, this synergy seems less efficient than in vitro, suggesting that the conditions of the treatment need to be optimized.

\section{Sensitivity of the mutants $X 47 \mathrm{hdp} A \Omega \mathrm{Km}$ and $X 47 \mathrm{mltD} \Omega \mathrm{Km}$ to bulgecin $A$}

From this study we postulated that the simultaneous targeting of different stages of the PG metabolism of $H$. pylori constitute a good therapeutic approach. Hence, we decided to test the activity of bulgecin A on mutants lacking PGrelated enzymes, as candidates for potential new synergies. We estimated the impact of bulgecin $\mathrm{A}$ on the mutants $\mathrm{X} 47 \mathrm{hdp} A \Omega \mathrm{Gm}$ and $\mathrm{X} 47 \mathrm{mltD} \Omega \mathrm{Km}$, by following the absorbance at $600 \mathrm{~nm}$, during $47 \mathrm{hr}$ (Fig. 7). These deletions were chosen for their absence of effect on bacterial growth, allowing a better estimation of their synergic potential with bulgecin $\mathrm{A}^{3}$ The results showed that bulgecin $\mathrm{A}$ induced a strong growth defect on the mutant $\mathrm{X} 47 \mathrm{~h} d p A \Omega \mathrm{Km}$, while $\mathrm{X} 47 \mathrm{mltD} \Omega \mathrm{Km}$ and the parental strain grew normally in the same conditions. This suggested that Slt and HdpA could have a direct functional link during PG biosynthesis.

\section{Discussion}

In the present study, we characterized the activity of bulgecin A on the PG metabolism of H. pylori. This compound was discovered in the 1980 s by a pharmaceutical company, which thought, at the time, to be working on a new $\beta$-lactam. ${ }^{13}$ Few years later, bulgecin A turned out to be an inhibitor of the LTG Slt70 in E. coli, responsible for bulges formation in presence of $\beta$-lactams. ${ }^{24}$ Indeed, Templin and his collaborators highlighted the increased sensitivity of the E. coli sltY mutant to $\beta$-lactams, while its growth was intact.
At the same time, they demonstrated the inhibitory activity of bulgecin A on Slt, by following, in different conditions, the activity of the purified protein on radio-labeled murein. Hence, they proposed as a mechanism of action that bulgecin A would act on Slt70 by competing for the PG binding domain, based on the composition of the molecule, that carries a GlcNAc, similar to those of the murein. However, the X-ray structure of Slt70 in complex with bulgecin A did not corroborate this hypothesis. Structural analysis indicated that bulgecin A was acting on Slt70 not only by interacting with the GlcNAc moiety, but also by forming a hydrogen bond between the predicted catalytic residues of Slt (Glu478), via the proline moiety of bulgecin A. Therefore, the authors proposed bulgecin A rather as an inhibitor of the active site of Slt70. ${ }^{25}$ Here we showed that bulgecin A was also able to inactivate the Slt70 homolog in H. pylori, mainly based on PG analysis. Interestingly, our data indicated that this inhibitory activity was specific to Slt, while the other LTG described in H. pylori, MltD, seemed unaffected. To better understand this observation, we performed docking analysis on both LTGs in presence of bulgecin A. Our models predicted that despite MltD and Slt would be formed by a large ring-shaped alphasuperhelix similarly to Slt70, only Slt would exhibit an extra alpha helix, close to the active site. Then, taking into account that MltD and Slt share strong similarities in terms of structure and catalytic domain residues (among them the putative active site), we postulated that the presence of this extra helix in Slt participates in the proper docking of bulgecin $\mathrm{A}$ and could explain why MltD is not affected by bulgecin A. Mutants targeting this extra helix are required to confirm this hypothesis.

Despite inactivating Slt, we showed that bulgecin A did not impact notably on the growth or on the morphology of H. pylori, as previously demonstrated in E. coli and Serratia
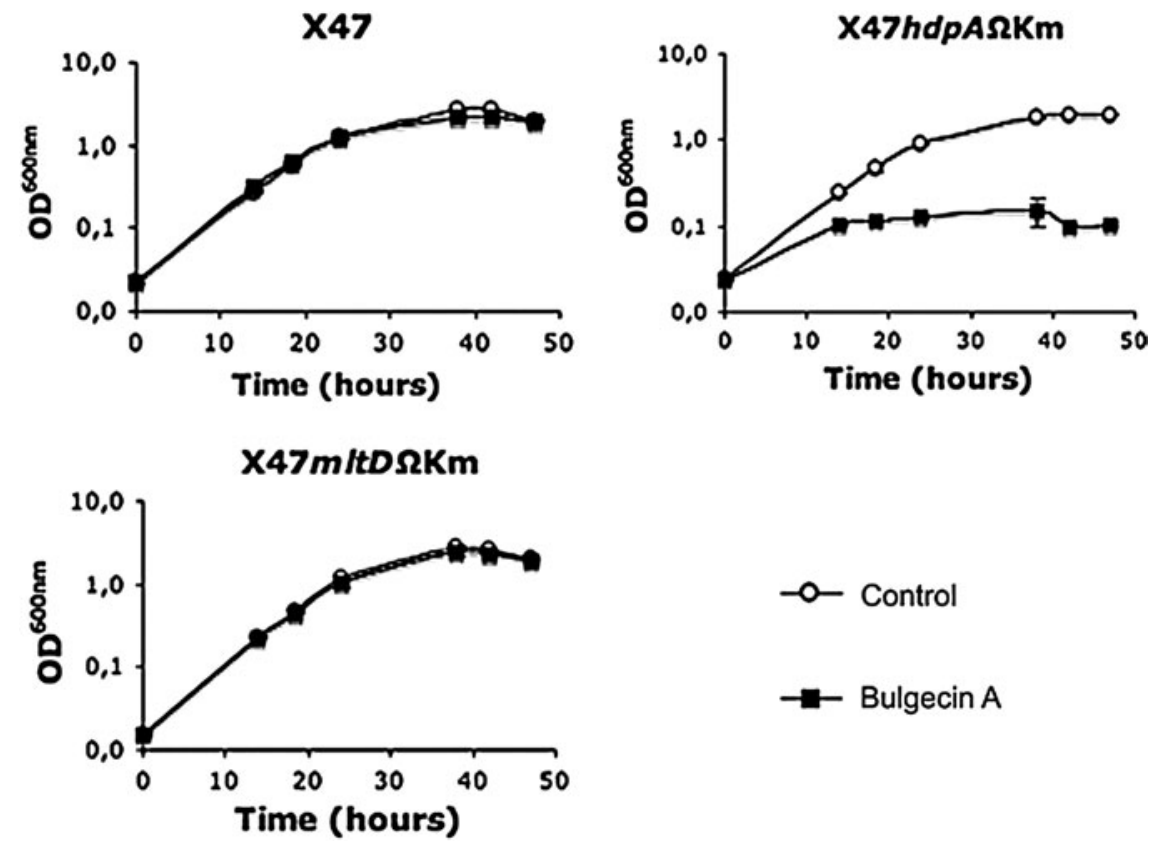

FIG. 7. Effect of bulgecin A on the growth of PG-related H. pylori mutants. X47 and the mutants $\mathrm{X} 47 \mathrm{mltd} \Omega \mathrm{Km}$ and $\mathrm{X} 47 \mathrm{hp} 0506 \Omega \mathrm{Km}$ were grown in liquid cultures in the presence or absence of bulgecin A $\left(100 \mu \mathrm{g} \cdot \mathrm{ml}^{-1}\right)$. Their growth was followed by measuring the OD ${ }^{600 \mathrm{~nm}}$ of triplicates for each condition, during $47 \mathrm{hr}$. 
marcescens. ${ }^{18}$ PG hydrolases and especially LTGs often have redundant functions in most bacteria. For example, 8 LTGs were described in E. coli, while only two were identified from the genome analysis of $H$. pylori. After the construction of the hexamutant MHD79 in E. coli, Heidrich et al. showed that this mutant grew similarly to the parental strain, but presented a reduced, but nonabolished, amount of anhydromuropeptides. $^{12}$ This latter point indicated that some LTGs were still unidentified at the time. Since, two additional LTGs were characterized in E. coli. ${ }^{1}$ Likewise, the double-mutant slt/mltD of $H$. pylori still presents anhydromuropeptides in its murein. ${ }^{5}$ In addition, we showed that bulgecin A had no impact on the growth of the mutant $\mathrm{X} 47 \mathrm{mlt} \mathrm{D} \Omega \mathrm{Km}$. Therefore, these observations could indicate that $H$. pylori possesses more than two LTG activities. It would be interesting to identify this/these enzyme(s), in particular with the aim to target the cell viability by inactivating simultaneously all of them. More investigations based on docking study of the bulgecin A binding to LTGs could indicate some ways of modifying this compound, to inactivate different LTGs at the same time.

In contrast, the mutant $\mathrm{X} 47 \mathrm{~h} d p A \Omega \mathrm{Km}$ exhibited a strong susceptibility to bulgecin A. HdpA carries both D,D-carboxy and -endopeptidases activities, usually a function of lowmolecular-weight PBPs, such as PBPs 4 to 8 in E. coli. Hence, $\mathrm{HdpA}$ has been proposed to participate in the regulation of the pool of the PBPs substrate-the GM pentapeptide, whose accumulation could lead to the emergence of branched cells when HdpA activity is abolished. ${ }^{3}$ Interestingly, when incubated with bulgecin A and mecillinam (a inhibitor of PBP2), S. marcescens forms not only bulges, but also branches, associated with an increased lethality. Additionally, Slt70 is able to interact with the low-molecular-weight PBP $7 / 8$ in vitro. ${ }^{19}$ Thus, the authors proposed the existence in vivo of an enzymatic complex formed by Slt70, the PBP7/8 and the PBP3. Therefore, our data coupled with previous work comforted the hypothesis of a functional link between Slt and HdpA in H. pylori, potentially via protein-protein interactions, which are under investigation.

Otherwise, we focused a part of this study on the characterization of the synergy between bulgecin A and amoxicillin. Indeed, by searching a way to potenciate the inhibitory activity of bulgecin $\mathrm{A}$, we discovered that its concomitant use with amoxicillin (inhibiting the PBPs) provoked strong damages to $H$. pylori in terms of cell viability and morphology. We reported that this synergy was reproducible in all the $H$. pylori strains tested (despite the high genetic polymorphism present in this species), including a strain that presents a reduced susceptibility to amoxicillin (strain S107). Since no spread of resistance to amoxicillin has been yet reported in $H$. pylori, this combination of molecules constitutes an attractive therapeutic approach against this infection. Moreover, bulgecin A is not toxic as previously predicted by Shinagawa et al., who estimated its $\mathrm{LD}_{50}$ for being superior to $1.0 \mathrm{mg} \mathrm{kg}{ }^{-1}$ by intravenous injection, in mice. ${ }^{21}$ Thus, we tested its effect on the $H$. pylori ability to colonize the mouse gastric mucosa. Our results revealed that in spite of presenting an attenuated activity, bulgecin A associated with amoxicillin induced a slight, but significant, decrease in colonization rate. This weak activity could be explained by a potential poor stability of the molecule in the mouse gastric mucosa. Indeed, the pharmacological properties of bulgecin
A in vivo are poorly described, and further investigations would be required to chemically modify bulgecin A to increase its potency in vivo.

As mentioned above, the synergy between bulgecin A and amoxicillin has previously been described in E. coli. Trying to understand the underlying mechanism, Kraft proposed that, by inactivating Slt70, bulgecin A would decrease the level of turnover products present in the cytoplasm, and consequently lead to the repression of ampC, coding for a $\beta$-lactamase. ${ }^{14}$ Few years later, Simm corroborated this hypothesis by showing that bulgecin A was able to inactivate the $\beta$-lactamase BceII from Bacillus cereus. ${ }^{22}$ Nevertheless, no $\beta$-lactamase has been rigorously described in H. pylori. ${ }^{26}$ Hence, in the case of this species, we could rather imagine that bulgecin A would affect the activity of high-molecular-weight PBPs, potentially by interfering with interaction between a PBP and Slt. Indeed, such interactions have been previously described in vitro, for example, between PBP3 and Slt70 in E. coli, and between SltB1 and PBP2 in Pseudomonas aeruginosa. ${ }^{16,19}$ Moreover, Legaree et al. showed that the morphological effects and lysis caused by the overproduction of the PBP2 in E. coli were abolished in the hexamutant MHD79. They proposed therefore that PBP2 and LTGs could interact in vivo through the formation of multienzyme complexes. ${ }^{15}$ Thus, a similar process could be possible in $H$. pylori, especially taking into account that the sub-inhibitory amount of amoxicillin used for the present study is predicted to inhibit preferentially PBP $2 .{ }^{6}$ Hence, we propose that Slt could interact with HdpA and PBP2 in a multienzyme complex, leading to an increased sensitivity of $H$. pylori to bulgecin A when either PBP2 or HdpA are inactivated.

To conclude, in addition to showing the attractive synergic potential of bulgecin A on $H$. pylori survival, our study provided new clues for the understanding of the PG metabolism.

\section{Acknowledgments}

We thank the Takeda Pharmaceutical Company Ltd. for providing bulgecin A. Mathilde Bonis was supported by a Ph.D. fellowship (Ministère de l'Enseignement Supérieur et de la Recherche, France) and by a Pasteur-Weizmann fellowship. Allison Williams was supported by a long-term EMBO fellowship. This study was funded in part by the ERC starting grant to Ivo G. Boneca (PGNfromSHAPEtoVIR no. 202283).

\section{Disclosure Statement}

No competing financial interests exist.

\section{References}

1. Artola-Recolons, C., L.I. Llarrull, E. Lastochkin, S. Mobashery, and J.A. Hermoso. 2011. Crystallization and preliminary X-ray diffraction analysis of the lytic transglycosylase MltE from Escherichia coli. Acta. Crystallogr. Sect. F Struct. Biol. Cryst. Commun. 67:161-163.

2. Boneca, I.G., C. Ecobichon, C. Chaput, A. Mathieu, S. Guadagnini, M.C. Prevost, F. Colland, A. Labigne, and H. de Reuse. 2008. Development of inducible systems to engineer conditional mutants of essential genes of Helicobacter pylori. Appl. Environ. Microbiol. 74:2095-2102. 
3. Bonis, M., C. Ecobichon, S. Guadagnini, M.C. Prevost, and I.G. Boneca. 2010. A M23B family metallopeptidase of $\mathrm{He}$ licobacter pylori required for cell shape, pole formation and virulence. Mol. Microbiol. 78:809-819.

4. Chaput, C., C. Ecobichon, N. Cayet, S.E. Girardin, C. Werts, S. Guadagnini, M.C. Prevost, D. Mengin-Lecreulx, A. Labigne, and I.G. Boneca. 2006. Role of AmiA in the morphological transition of Helicobacter pylori and in immune escape. PLoS Pathog. 2:e97.

5. Chaput, C., A. Labigne, and I.G. Boneca. 2007. Characterization of Helicobacter pylori lytic transglycosylases Slt and MltD. J. Bacteriol. 189:422-429.

6. DeLoney, C.R., and N.L. Schiller. 1999. Competition of various beta-lactam antibiotics for the major penicillinbinding proteins of Helicobacter pylori: antibacterial activity and effects on bacterial morphology. Antimicrob. Agents Chemother. 43:2702-2709.

7. El Ghachi, M., P.J. Mattei, C. Ecobichon, A. Martins, S. Hoos, C. Schmitt, F. Colland, C. Ebel, M.C. Prevost, F. Gabel, P. England, A. Dessen, and I.G. Boneca. 2011. Characterization of the elongasome core PBP2: MreC complex of Helicobacter pylori. Mol. Microbiol. 82:68-86.

8. Ermak, T.H., P.J. Giannasca, R. Nichols, G.A. Myers, J. Nedrud, R. Weltzin, C.K. Lee, H. Kleanthous, and T.P. Monath. 1998. Immunization of mice with urease vaccine affords protection against Helicobacter pylori infection in the absence of antibodies and is mediated by MHC class IIrestricted responses. J. Exp. Med. 188:2277-2288.

9. Ferrero, R.L., V. Cussac, P. Courcoux, and A. Labigne. 1992. Construction of isogenic urease-negative mutants of Helicobacter pylori by allelic exchange. J. Bacteriol 174:42124217.

10. Glauner, B. 1988. Separation and quantification of muropeptides with high-performance liquid chromatography. Anal. Biochem. 172:451-464.

11. Hayashi, K. 1975. A rapid determination of sodium dodecyl sulfate with methylene blue. Anal. Biochem. 67:503-506.

12. Heidrich, C., A. Ursinus, J. Berger, H. Schwarz, and J.V. Holtje. 2002. Effects of multiple deletions of murein hydrolases on viability, septum cleavage, and sensitivity to large toxic molecules in Escherichia coli. J. Bacteriol. 184:6093-6099.

13. Imada, A., K. Kintaka, M. Nakao, and S. Shinagawa. 1982. Bulgecin, a bacterial metabolite which in concert with betalactam antibiotics causes bulge formation. J. Antibiot. (Tokyo) 35:1400-1403.

14. Kraft, A.R., J. Prabhu, A. Ursinus, and J.V. Holtje. 1999. Interference with murein turnover has no effect on growth but reduces beta-lactamase induction in Escherichia coli. J. Bacteriol. 181:7192-7198.

15. Legaree, B.A., C.B. Adams, and A.J. Clarke. 2007. Overproduction of penicillin-binding protein 2 and its inactive variants causes morphological changes and lysis in Escherichia coli. J. Bacteriol. 189:4975-4983.

16. Legaree, B.A., and A.J. Clarke. 2008. Interaction of penicillinbinding protein 2 with soluble lytic transglycosylase B1 in Pseudomonas aeruginosa. J. Bacteriol. 190:6922-6926.

17. Marshall, B.J., D.B. McGechie, P.A. Rogers, and R.J. Glancy. 1985. Pyloric Campylobacter infection and gastroduodenal disease. Med. J. Aust. 142:439-444.

18. Nakao, M., K. Yukishige, M. Kondo, and A. Imada. 1986. Novel morphological changes in gram-negative bacteria caused by combination of bulgecin and cefmenoxime. Antimicrob. Agents Chemother. 30:414-417.

19. Romeis, T., and J.V. Holtje. 1994. Specific interaction of penicillin-binding proteins 3 and $7 / 8$ with soluble lytic transglycosylase in Escherichia coli. J. Biol. Chem. 269:2160321607.

20. Roure, S. 2010. Rôle des Transglycosylases Lytiques Dans la Mobilité Chez Helicobacter pylori. Thesis Paris VI, Paris.

21. Shinagawa, S., M. Maki, K. Kintaka, A. Imada, and M. Asai. 1985. Isolation and characterization of bulgecins, new bacterial metabolites with bulge-inducing activity. J. Antibiot. (Tokyo) 38:17-23.

22. Simm, A.M., E.J. Loveridge, J. Crosby, M.B. Avison, T.R. Walsh, and P.M. Bennett. 2005. Bulgecin A: a novel inhibitor of binuclear metallo-beta-lactamases. Biochem. J. 387:585-590.

23. Sycuro, L.K., Z. Pincus, K.D. Gutierrez, J. Biboy, C.A. Stern, W. Vollmer, and N.R. Salama. 2010. Peptidoglycan crosslinking relaxation promotes Helicobacter pylori's helical shape and stomach colonization. Cell 141:822-833.

24. Templin, M.F., D.H. Edwards, and J.V. Holtje. 1992. A murein hydrolase is the specific target of bulgecin in Escherichia coli. J. Biol. Chem. 267:20039-20043.

25. Thunnissen, A.M., H.J. Rozeboom, K.H. Kalk, and B.W. Dijkstra. 1995. Structure of the 70-kDa soluble lytic transglycosylase complexed with bulgecin A. Implications for the enzymatic mechanism. Biochemistry 34:1272912737.

26. Tomb, J.F., O. White, A.R. Kerlavage, R.A. Clayton, G.G. Sutton, R.D. Fleischmann, et al. 1997. The complete genome sequence of the gastric pathogen Helicobacter pylori. Nature 388:539-547.

27. van Asselt, E.J., A.M. Thunnissen, and B.W. Dijkstra. 1999. High resolution crystal structures of the Escherichia coli lytic transglycosylase Slt70 and its complex with a peptidoglycan fragment. J. Mol. Biol. 291:877-898.

28. Viala, J., C. Chaput, I.G. Boneca, A. Cardona, S.E. Girardin, A.P. Moran, R. Athman, S. Memet, M.R. Huerre, A.J. Coyle, P.S. DiStefano, P.J. Sansonetti, A. Labigne, J. Bertin, D.J. Philpott, and R.L. Ferrero. 2004. Nod1 responds to peptidoglycan delivered by the Helicobacter pylori cag pathogenicity island. Nat. Immunol. 5:1166-1174.

29. Werts, C., L. le Bourhis, J. Liu, J.G. Magalhaes, L.A. Carneiro, J.H. Fritz, S. Stockinger, V. Balloy, M. Chignard, T. Decker, D.J. Philpott, X. Ma, and S.E. Girardin. 2007. Nod1 and Nod2 induce CCL5/RANTES through the NF-kappaB pathway. Eur. J. Immunol. 37:2499-2508.

30. Wroblewski, L.E., R.M. Peek, Jr., and K.T. Wilson. 2010. Helicobacter pylori and gastric cancer: factors that modulate disease risk. Clin. Microbiol. Rev. 23:713-739.

Address correspondence to: Ivo G. Boneca, Ph.D.

Group Biology and Genetics of the Bacterial Cell Wall

28 me du Dr. Roux Institut Pasteur 75724 Paris

France

E-mail: bonecai@pasteur.fr 